\title{
Constraining Cosmological Parameters with Image Separation STATiSTiCs OF GravitaTionally LENSED SDSS QuaSARS: MEAN IMAGE SEPARATION AND LIKELIHOOD INCORPORATING LENS GALAXY BRIGHTNESS
}

\author{
Du-Hwan Han and MyeOng-Gu Park \\ Department of Astronomy and Atmospheric Sciences, Kyungpook National University, Daegu, 702-701, Korea \\ mgp@knu.ac.kr \\ Received February 9, 2015; accepted February 17, 2015
}

\begin{abstract}
Recent large scale surveys such as Sloan Digital Sky Survey have produced homogeneous samples of multiple-image gravitationally lensed quasars with well-defined selection effects. Statistical analysis on these can yield independent constraints on cosmological parameters. Here we use the image separation statistics of lensed quasars from Sloan Digital Sky Survey Quasar Lens Search (SQLS) to derive constraints on cosmological parameters. Our analysis does not require knowledge of the magnification bias, which can only be estimated from the detailed knowledge on the quasar luminosity function at all redshifts, and includes the consideration for the bias against small image separation quasars due to selection against faint lens galaxy in the follow-up observations for confirmation. We first use the mean image separation of the lensed quasars as a function of redshift to find that cosmological models with extreme curvature are inconsistent with observed lensed quasars. We then apply the maximum likelihood test to the statistical sample of 16 lensed quasars that have both measured redshift and magnitude of lens galaxy. The likelihood incorporates the probability that the observed image separation is realized given the luminosity of the lens galaxy in the same manner as Im et al. (1997). We find that the $95 \%$ confidence range for the cosmological constant (i.e., the vacuum energy density) is $0.72 \leq \Omega_{\Lambda} \leq 1.0$ for a flat universe. We also find that the equation of state parameter can be consistent with -1 as long as the matter density $\Omega_{m} \lesssim 0.4$ (95\% confidence range). We conclude that the image separation statistics incorporating the brightness of lens galaxies can provide robust constraints on the cosmological parameters.
\end{abstract}

Key words: gravitational lensing — lensing statistics — cosmology — cosmological constant — galaxies - galaxy parameters

\section{INTRODUCTION}

A small fraction of quasars are gravitationally lensed into multiple images by a foreground galaxy. The angular separation between the multiple images is determined by the bending angle and the angular diameter distances to the source quasar and the lens galaxy. The bending angle is determined by the mass distribution of the lens galaxy and the angular diameter distance by the geometry and the evolution of the universe (see e.g., Schneider et al. 1992). Therefore, the angular separation of gravitationally lensed quasars carry valuable information on the lens galaxies and the cosmology. The probability that a given quasar is multiply imaged by gravitational lensing is also determined by the combination of the lensing cross-section of each lens galaxy and the total number of lens galaxies out to the source redshift.

Hence, the first discovery of the multiple image quasar lens system, Q0957+561, by Walsh et al. (1979) and subsequent discoveries of the similar lens systems led to the theory of the statistical properties of multiple image lensed quasar systems, especially the statis-

Corresponding Author: M.-G. Park tics of the image separations and the lensing probability (Turner et al. 1984), which can be applied to the cosmological test. The first application of the image separation statistics was the mean image separation versus redshift test, i.e., to check how the mean image separation varies as a function of source (quasar) redshift (Gott et al. 1989, GPL hereafter). If the galaxies are approximated by the singular isothermal spheres (SIS) and uniformly distributed in the universe, the mean separation should remain constant in the flat universe as the source redshift varies, regardless of the magnitude of the cosmological constant in the FriedmannLemaitre-Robertson-Walker (FLRW) universe. Unfortunately, the number of observed lensed quasars was too small at the time and the image separations observed had too large variations to reliably constrain the geometry of the universe. Lee \& Park (1994) subsequently calculated the distribution of angular image separation for lensed quasars by considering appropriate observational selection effects and adopting an assumption that galaxies are SISs that follow Schechter luminosity function to compare with the observed image separations, and found that cosmological models with a large cosmological constant is preferred albeit 
with limited statistical significance. Park (1996) also checked if the observed image separation distribution is compatible with that from the "standard lens statistics model', a flat FLRW universe with homogeneously distributed SIS galaxies, only to find possible incompatibilities. Park \& Gott (1997, PG97) revisited the mean image separation test: they compiled then-available lensed quasar systems and found that the mean image separation decreases as the source redshift increases. This decrease is expected in an open universe, but the decrease as a function of source redshift was much faster than even that expected in an empty universe. Diverse galaxy model profile or lensing aided by the cluster could not explain this steep decrease, and PG97 wondered if there are any unknown selection effects. Diverse solutions have been suggested: a logarithmic surface mass profile of lens galaxy (Williams 1997) or an inhomogeneous universe (Helbig 1998). Or it could be that some quasar pairs are not gravitationally lensed ones (Kochanek 1999). Helbig (1998) also showed that when another lens case or JVAS/CLASS radio selected cases are added to the lens sample used by PG97, the statistical test result becomes different: most cosmological models are not rejected at 95\% confidence level. This illustrated that how the lens sample is constructed can affect the outcome of the lensing test. Therefore, a large number of lens samples from homogeneous survey that has a well-defined selection effect is required for meaningful cosmological test.

Fukugita \& Turner (1991) and Fukugita et al. (1992) instead utilized the lensing probability because it sensitively depends on the cosmological constant. They compared the number of discovered lensed quasars against the known catalog of quasars to estimate the observed probability, and concluded that the cosmological constant cannot be too large, $\Omega_{\Lambda}<0.9$. Otherwise, we should be able to observe an order-of-magnitude larger number of lensed quasars.

Maoz \& Rix (1993) extended the analysis to utilize both image separation and the lensing probability: they compared the expected number of lensed quasars as a function of the image separations with the number distribution of known lensed quasars within the HST snapshot sample. But they found that the halo of the lens galaxy rather than the cosmological model was constrained better. Kochanek (1996) tried more comprehensive lensing test. He used maximum likelihood test that incorporated as much information from lensed quasars, such as number of images, redshifts, magnitudes, and separations, as possible and compared with the expected probability distribution in the standard cosmological models with constant comoving density of non-evolving $\mathrm{E} / \mathrm{SO}$ galaxies to put constraints on the cosmological constant, which yielded $\Omega_{\Lambda}<0.66$ at $95 \%$ confidence level. However, further similar studies by Chiba \& Yoshii $(1997,1999)$ with updated parameters for lens galaxies and QSOs concluded that a flat universe with large $\Omega_{\Lambda}$ was compatible with the lensed QSO data. Im et al. (1997) used multiple image QSO systems lensed by elliptical galaxies to measure $\Omega_{\Lambda}$.
They, unlike previous studies, used additionally the redshift and the magnitude of the lens galaxies to infer the velocity dispersion of the lens galaxy through FaberJackson relation, thereby fixing all lensing-related parameters of the lens galaxies. They also found that a flat universe with large $\Omega_{\Lambda}$ is preferred and a flat universe with the matter-density parameter $\Omega_{m}=1$ is rejected at $97 \%$ confidence level. Chae (2003) later applied probability test to better defined radio selected Cosmic Lens All-Sky Survey (CLASS) sample to deduce $\Omega_{m}=0.31_{-0.14}^{+0.27}$ ( $68 \%$ confidence). Hence, the outcome of the early lensing tests seems to contradict that of the later tests.

These apparently contradictory outcomes illustrate both the basic difficulties and the fragile nature of the cosmological lensing test. The light is bent by the gravitational field, i.e., the spacetime curvature, and the spacetime curvature contains contributions by the local mass distribution such as lens galaxy as well as that by the whole universe. If the local mass distribution is poorly determined, as is often the case with galaxies that harbor certain amount of dark matter, the cosmological lensing test may not produce correct constraints on the cosmological models. In addition, small number statistics on haphazardly discovered lens cases with non-uniform observational selection criteria can significantly distort the outcome of the statistical test.

Especially, the lensing probability should be corrected for the magnification bias to be properly compared with the actual observed lens cases. For a given magnitude-limited QSO survey, QSOs which are originally too dim to be detected within the survey can enter the survey sample when they are magnified enough by lensing (see e.g., Fukugita \& Turner 1991). Correct estimates of this magnification bias requires the knowledge of the original luminosity function of QSOs at all redshifts in addition to the mass distribution of lens galaxies at all redshifts since the magnification depends on the redshifts of source and lens as well as the mass of the lens. The luminosity function of high redshift QSOs are still poorly determined and that of the radio sources are even worse, and therefore the magnification bias is not easily estimated.

The advent of carefully designed large scale redshift survey can be the solution to all these problems. For example, Sloan Digital Sky Survey (SDSS; York et al. 2000; Ahn et al. 2014 and references therein) has applied well-defined observational criteria to obtain by now five band photometry of 1.2 billion cataloged objects and spectra of 1.8 million galaxies and 0.3 million quasars (Data Release 10, Ahn et al. 2014). This will better determine the luminosity function of lens galaxies and QSOs, which will make the cosmological lensing test more reliable. Oguri et al. (2006) and Inada et al. (2010) conducted Sloan Digital Sky Survey Quasar Lens Search (SQLS) to search for gravitationally lensed multiple image quasars from the SDSS spectroscopic quasar catalogs (Schneider et al. 2005, 2007) by applying redefined selection procedures to find candidate lensed quasars and confirming them by follow-up observations. 
Table 1

List of lensed quasar systems from SQLS (Statistical Sample)

\begin{tabular}{lcccl}
\hline Objects & $z_{s}$ & $z_{l}$ & $\Delta \theta\left(^{\prime \prime}\right)$ & Reference \\
\hline SDSS J0746+4403 & 1.998 & 0.513 & 1.079 & Inada et al. (2007), Inada et al. (2010), Kayo et al. (2010) \\
SDSS J0806+2006 & 1.537 & 0.573 & 1.403 & Inada et al. (2006) \\
SDSS J0913+5259 & 1.377 & 0.830 & 1.140 & Oscoz et al. (1997) \\
SDSS J0924+0219 & 1.524 & 0.394 & 1.780 & Inada et al. (2003), Eigenbrod et al. (2006a) \\
SDSS J0951+2635 & 1.246 & 0.260 & 1.101 & Schechter et al. (1998), Eigenbrod et al. (2007) \\
SDSS J1021+4913 & 1.720 & 0.451 & 1.140 & Pindor et al. (2006) \\
SDSS J1055+4628 & 1.249 & 0.388 & 1.150 & Kayo et al. (2010) \\
SDSS J1251+2935 & 0.802 & 0.410 & 1.790 & Kayo et al. (2007) \\
SDSS J1313+5151 & 1.875 & 0.194 & 1.240 & Ofek et al. (2007) \\
SDSS J1332+0347 & 1.445 & 0.191 & 1.140 & Morokuma et al. (2007) \\
SDSS J1335+0118 & 1.571 & 0.440 & 1.560 & Oguri et al. (2004), Eigenbrod et al. (2006b) \\
SDSS J1353+1138 & 1.624 & 0.250 & 1.410 & Inada et al. (2006) \\
SDSS J1405+0959 & 1.810 & 0.660 & 1.980 & Jackson et al. (2012) \\
SDSS J1455+1447 & 1.424 & 0.420 & 1.730 & Kayo et al. (2010) \\
SDSS J1515+1511 & 2.054 & 0.742 & 1.950 & Inada et al. (2012) \\
SDSS J1524+4409 & 1.210 & 0.320 & 1.669 & Oguri et al. (2008) \\
\hline
\end{tabular}

a Two lens systems are already known lensed QSO systems. SDSS J0913 + 5259 and SDSS J0951 + 2635 are known the lensed QSO systems by SBS0909+ 523 and FBQ0951 + 2635, respectively.

Likelihood analysis (Kochanek 1993) on SQLS sample to test the probability of a observed lensed quasar to have a given redshift and image separation for a flat universe (Oguri et al. 2012) yielded improved constraints on the cosmological constant, $\Omega_{\Lambda}=0.79_{-0.07}^{+0.06}$ (68\% confidence, statistical error only), which can serve as an independent check on cosmology in addition to the constraints from SN Ia (Perlmutter et al. 1997; Riess et al. 1998), CMBR (Komatsu et al. 2009), and large scale structure analysis (Percival et al. 2007).

However, we worry that certain uncertainties may exist in the number and luminosity evolutions of the galaxies and quasars since current large scale surveys cover mainly low-redshift universe while lensing occurs on high redshift quasars and galaxies. This can introduce non-trivial systematic errors. Therefore, Han \& Park (2014) applied only the image separation statistics, which is free from magnification bias, to the cosmological model with SQLS sample. They used the relative or normalized probability for a lensed quasar to have the observed image separation given that a multiple image lensing occurred between a quasar at the source redshift $z_{s}$ and a galaxy at the lens redshift $z_{l}$. This is a posteriori probability that does not require the knowledge on the density and luminosity distribution of quasars as a function of $z_{s}$ nor the density distribution of lens galaxies as a function of $z_{l}$. Only the probability distribution of the velocity dispersion of lens galaxies approximated by SISs at a given redshift is required. The resulting constraints for the universe with cosmological constant were roughly perpendicular to the $k=0$ line in $\Omega_{m}-\Omega_{\Lambda}$ plane similar to the SN Ia constraints, but wide enough to include most flat universe models, much wider than theoretically expected (Han \& Park 2014).

In this work, we use additional analyses on SQLS sample that utilize the image separation statistics: mean image separation as a function of the quasar redshift (PG97) and the image separation probability that also incorporates the luminosity of lens galaxies (Im et al. 1997). We test for galaxy properties as well as cosmology. In Section 2, we explain the sample we use. In Section 3, relevant theory of lensing statistics is laid out, and in Section 4, we summarize the work.

Throughout the paper, we assume the dimensionless Hubble constant $h \equiv H_{0} / 100 \mathrm{~km} \mathrm{sec}^{-1} \mathrm{Mpc}^{-1}=0.7$.

\section{Data Sample and Basic Assumptions}

We use SQLS lens systems based on the SDSS DR7 quasar catalog (Schneider et al. 2010; Oguri et al. 2006; Inada et al. 2012). Inada et al. (2012) provides 62 lensed quasar systems that consist of the statistical sample and the additional sample: statistical sample satisfies the completeness condition and the redshift, magnitude, and image separation ranges defined for statistical analysis while the additional sample does not satisfy them. Our statistical sample listed in Table 1 consists of 16 systems lensed by a single galaxy with reliably measured redshift among the SQLS statistical sample (Inada et al. 2012). The details of lens sample are given in Han \& Park (2014). For the mean image separation test, we only need quasar redshift. Hence, we use whole 38 lensed quasar systems that include 16 statistical lens systems and 22 additional lens systems from the SQLS additional sample (Inada et al. 2012) that satisfy our angular selection criterion (Han \& Park 2014). Crosses and circles in Figure 1a show the maximum angular image separation of our sample quasar systems as a function of a source quasar redshift, respectively. The distribution does not show any obvious strong decrease of image separation in redshift as noted and analyzed by PG97, largely due to the addition of wide separation quasars at high redshifts and $4^{\prime \prime}$ upper limit of SQLS sample. 

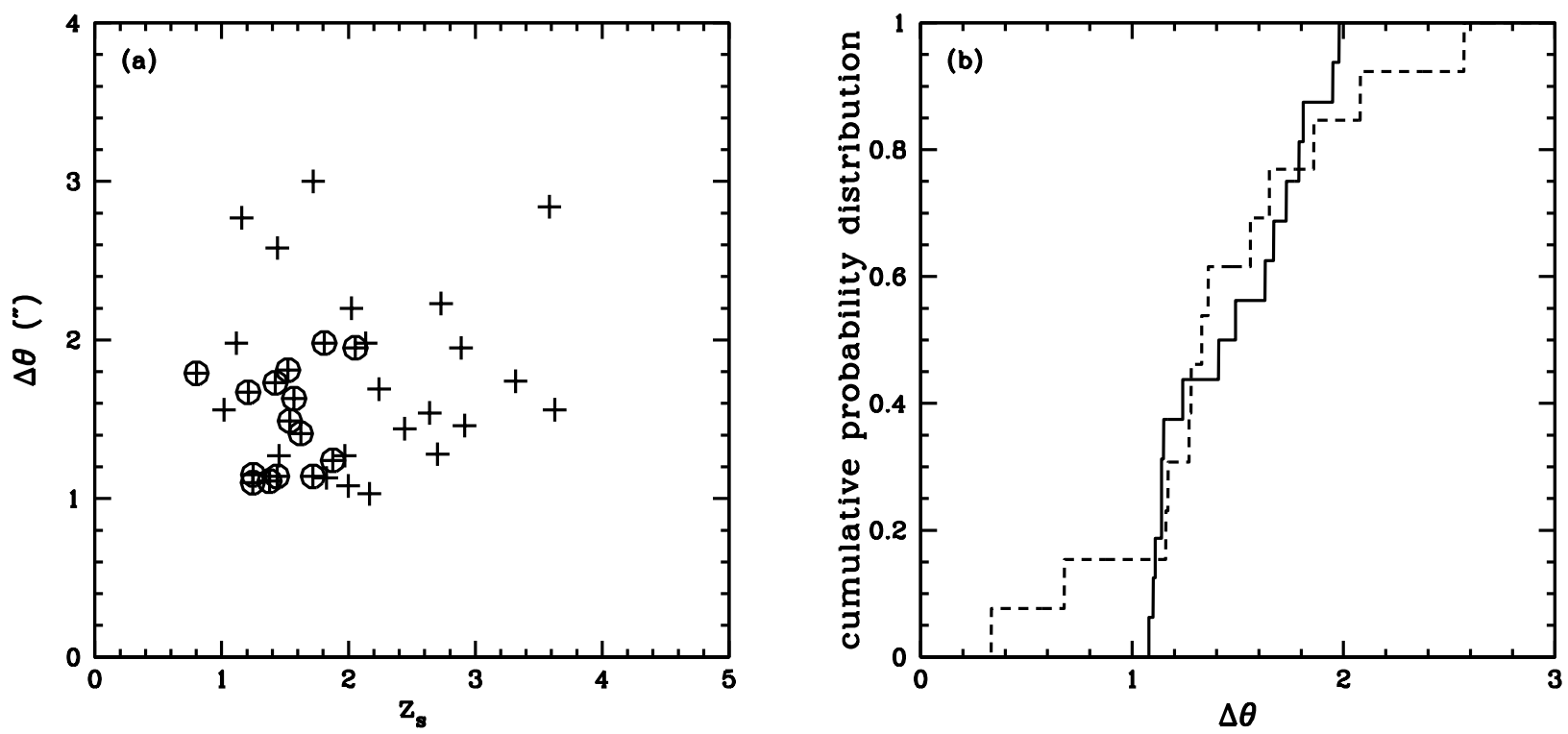

Figure 1. (a) Maximum image separation $\Delta \theta$ versus source redshift $z_{s}$ of whole 38 SQLS quasar lens systems (cross) and 16 statistical lens systems (circle). (b) The cumulative probability distribution of the our statistical lens sample (solid line) and the JVAS/CLASS QSO lens sample (dashed line) for comparison.

Before SQLS, the Jodrell Bank-VLA Astrometric Survey/Cosmic Lens All Sky Survey (JVAS/CLASS: Myers et al. 2003; Browne et al. 2003) was the largest homogeneous lens sample suitable for statistical analysis (Chae 2003). Because the statistical test depends sensitively on the lens sample used, we check if our statistical lens sample is any different from JVAS/CLASS sample. Figure 1b shows the cumulative probability distribution function in image separation of our statistical lens sample (solid line) and JVAS/CLASS sample (dashed line). JVAS/CLASS sample spans wider image separation range due to higher angular resolution and dynamic range of radio survey over optical survey. The Kolmogorov-Smirnov test, however, yields the probability to have the maximum difference seen between the two samples is 0.83 . This means the null hypothesis that the two samples are drawn from the same distribution is not rejected; there is no significant statistical difference between the two samples.

We use the same cosmological models and physical parameters regarding lens galaxies as in Han \& Park (2014). We assume FLRW universe. Lens galaxies are approximated by singular isothermal spheres (SISs) whose velocity dispersion distribution (VDF) is determined by the SDSS galaxy samples (Sheth et al. 2003; Choi et al. 2007; Chae 2010). The probability of multiple image lensing is also given in Han \& Park (2014).

In the image separation statistics test, the most important observational selection effect is the angular selection effect on the sample. SDSS has a well-defined photometric and spectroscopic selection effects and the search algorithm for SQLS is also carefully defined (Oguri et al. 2006; Inada et al. 2012), and the angular selection effect can be reliably determined (Han \& Park 2014). However, there is more subtle angular selection effect in SQLS. The follow-up observations for SQLS have a limiting apparent $I$-band magnitude of 23 for the lens galaxy (Inada et al. 2010). Because galaxy's luminosity is related to the velocity dispersion (FaberJackson relation or Tully-Fisher relation), selection effect against dim lens galaxies result in bias against lensing by low velocity-dispersion galaxies, therefore, small image separation systems. The details of the angular selection effect from this limit on the lens magnitude are given in Han \& Park (2014).

\section{Statistical Tests}

\subsection{Mean Image Separation and The Curvature Test}

GPL have shown that the mean image separation does not vary with source redshift if the FLRW universe is flat regardless of the value of $\Omega_{\Lambda}$, and the variation of the mean image separation as a function of the source quasar redshift can be used to test the curvature of the universe (GPL; PG97). The mean image separation, $\langle\Delta \theta\rangle$, in the presence of the angular selection effect, either from direct angular selection or from indirect selection bias due to limiting magnitude on the lens, is

$$
\begin{aligned}
\langle\Delta \theta\rangle= & 8 \pi\left(\frac{\sigma^{*}}{c}\right)^{2} \\
& \times \frac{\int_{0}^{z_{s}} A\left(\frac{D_{l s}}{D_{o s}}\right)^{3} \Gamma\left(\frac{6+\alpha}{\beta}, x\left(z_{l}\right)\right) \mathrm{d} z_{l}}{\int_{0}^{z_{s}} A\left(\frac{D_{l s}}{D_{o s}}\right)^{2} \Gamma\left(\frac{4+\alpha}{\beta}, x\left(z_{l}\right)\right) \mathrm{d} z_{l}}
\end{aligned}
$$



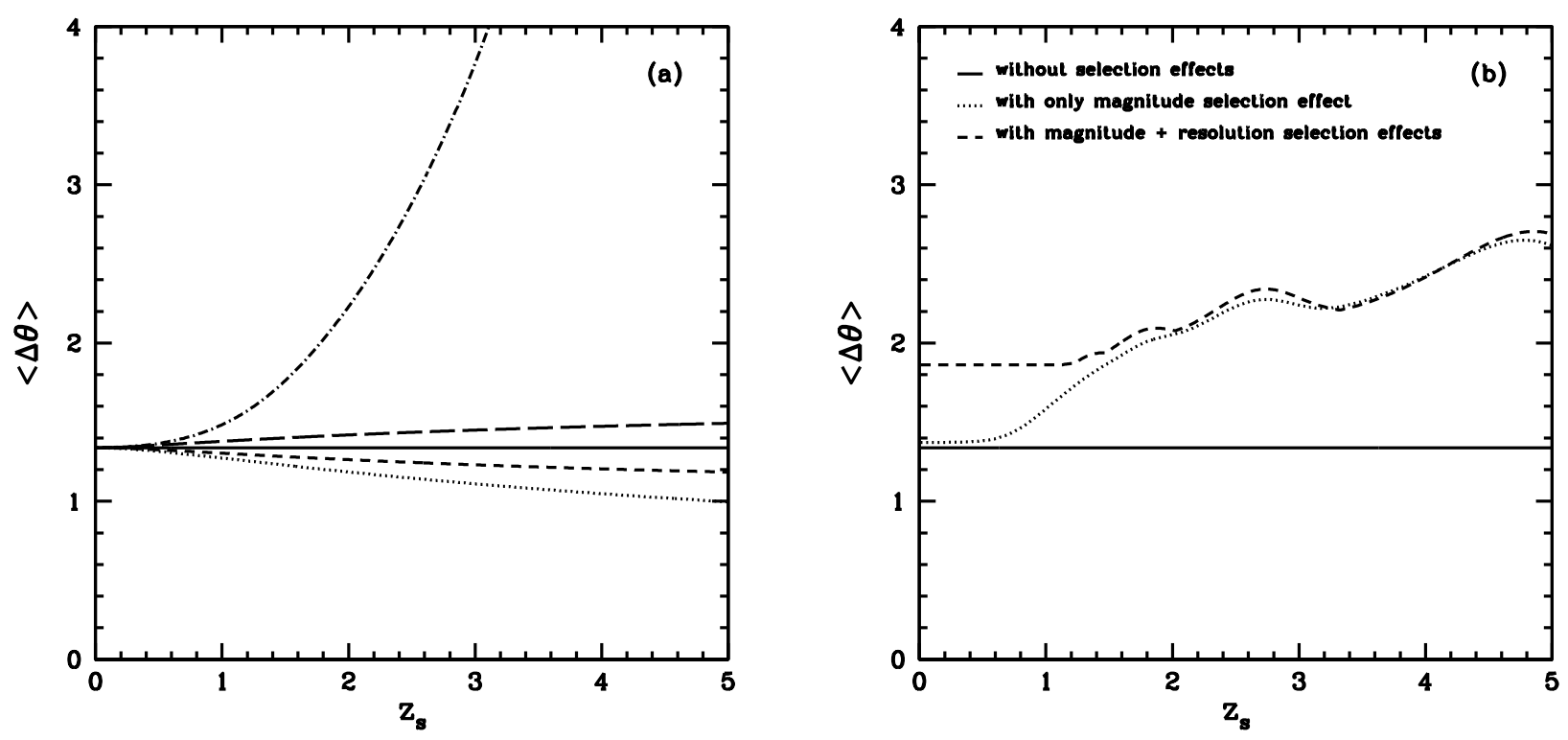

Figure 2. (a) The mean image separations as a function of source redshift without selection effects for highly closed universe $\left(\Omega_{m}=0.2, \Omega_{\Lambda}=1.43\right.$; dot-dashed line $)$, mildly closed universe $\left(\Omega_{m}=2.0, \Omega_{\Lambda}=0\right.$; long-dashed line $)$, flat universe $\left(\Omega_{m}+\Omega_{\Lambda}=1\right.$; solid line), mildly open universe $\left(\Omega_{m}=0.4, \Omega_{\Lambda}=0\right.$; dashed line $)$, and completely empty universe $\left(\Omega_{m}=0\right.$, $\Omega_{\Lambda}=0$; dotted line). (b) The mean image separations for a flat universe $\left(\Omega_{m}+\Omega_{\Lambda}=1\right)$ without selection effects (solid line), with only magnitude selection effect (dotted line), with magnitude and angular resolution selection effects (dashed line).

where

$$
\begin{aligned}
A= & \left(1+z_{l}\right)^{2} f\left(z_{l}\right) D_{o l}^{2}, \\
f\left(z_{l}\right)= & {\left[\Omega_{m}\left(1+z_{l}\right)^{3}+\left(1-\Omega_{m}-\Omega_{\Lambda}\right)\left(1+z_{l}\right)^{2}+\Omega_{\Lambda}\right]^{-1 / 2}, } \\
x\left(z_{l}\right) \equiv & {\left[\frac{1}{8 \pi} \frac{D_{o s}}{D_{l s}}\left(\frac{c}{\sigma_{*}}\right)^{2}\right]^{\frac{\beta}{2}} \Delta \theta_{\text {cut }}^{\beta / 2}, } \\
& \Gamma(a, x)=\int_{x}^{\infty} t^{a-1} e^{-t} d t,
\end{aligned}
$$

and $\Delta \theta_{\text {cut }}$ is the minimum separation of images due either to the angular selection or to the magnitude selection (Han \& Park 2014).

We reproduce $\langle\Delta \theta\rangle\left(z_{s}\right)$ without selection effect for five cosmological models in Figure 2a. If observations are perfect with no angular selection effect, for a flat universe we expect the mean image separation of approximately $1.35^{\prime \prime}$ at any $z_{s}\left(\Omega_{m}+\Omega_{\Lambda}=1\right.$; solid line). The mean image separations of lens sample (Figure 1 ) also do not show any obvious variation with $z_{s}$ considering rather large dispersion in the image separation. This is rather different from the sample analyzed by PG97: the discrepancy probably shows the importance and difficulty of the well-defined sample with enough numbers (Helbig 1998). However, the mean separations are mostly between $1.5^{\prime \prime}$ and $2.0^{\prime \prime}$, which is significantly larger than the expected value of $1.35^{\prime \prime}$. The selection effects due to the limiting angular resolution and lens magnitude are largely responsible for the difference.

In a completely empty universe $\left(\Omega_{m}=0, \Omega_{\Lambda}=0\right.$; dotted line) and mildly open universe $\left(\Omega_{m}=0.4\right.$, $\Omega_{\Lambda}=0$; dashed line), the mean image separations decrease as the source redshift increases from 0 to 5 . For a mildly closed universe, the mean image separations increase in the same source redshift interval $\left(\Omega_{m}=2.0\right.$, $\Omega_{\Lambda}=0$; long-dashed line). In a highly closed universe, the mean image separation can diverge as the source redshift increases $\left(\Omega_{m}=0.2, \Omega_{\Lambda}=1.43\right.$; dot-dashed line).

Figure $2 \mathrm{~b}$ shows the mean image separation for a flat universe when only the lens magnitude limit is considered (dotted line) and when both the angular resolution limit and the lens magnitude limit are considered (dashed line). At low redshifts $\left(z_{s}<0.6\right)$, the selection effect due to limiting angular resolution is more important, which increases the mean image separation to $1.85^{\prime \prime}$, in agreement with the observed mean image separations (Figure 1a). At high redshifts $\left(z_{s}>0.6\right)$, the selection effect due to the limiting magnitude for the lens galaxy becomes more important as expected and the mean image separation increases with $z_{s}$. For $z_{s}=5$, the mean image separation can be as large as $3^{\prime \prime}$. Hence, whenever there exists a magnitude limit for the lens galaxy either from candidate search processes or from follow-up observations for confirmation, this bias should be considered. The undulations at large redshift arises from the K-correction.

We now compare the observed mean image separation against the expected one to test the curvature of the universe: In a universe with given mean expected image separation $\langle\Delta \theta\rangle\left(z_{s}\right)$, the observed image separation divided by the mean expected separation, $\theta_{i} /\langle\Delta \theta\rangle\left(z_{s}\right)$, should be uncorrelated with the redshift $z_{s}$ (PG97). We test for this correlation with Spearman rank correlation test. For this test, we use whole 38 systems as mentioned in Section 2 (crosses in Figure 1a). 


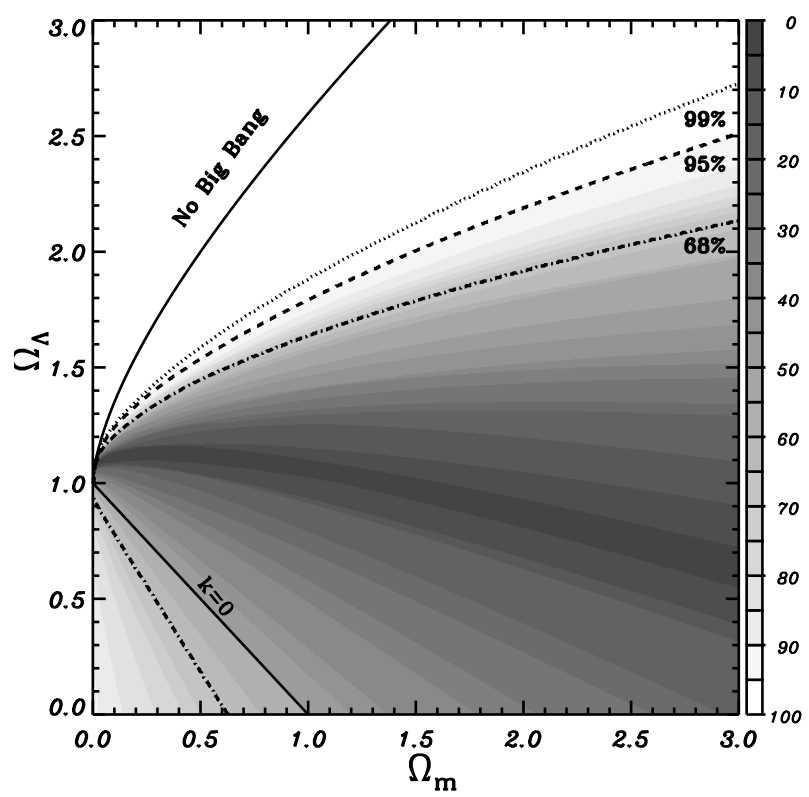

Figure 3. The contours of the probability calculated by the Spearman rank correlation test for the curvature of the universe. Dashed line is a region of non Big Bang, and longdashed line shows the flat universe.

In an empty universe $\left(\Omega_{m}=0, \Omega_{\Lambda}=0\right)$, the Spearman test shows that the probability of correlation shown in the sample produced by chance is $P=0.34$. This probability means that if our universe is really an empty one, the probability of observing such correlation by chance is $34 \%$. For a mildly open universe $\left(\Omega_{m}=0.4\right.$, $\Omega_{\Lambda}=0$ ), the Spearman test shows that the probability of correlation is $P=0.43$. Similarly, $P=0.78$ for a mildly closed universe $\left(\Omega_{m}=2.0, \Omega_{\Lambda}=0\right)$. But for a highly closed universe $\left(\Omega_{m}=0.2, \Omega_{\Lambda}=1.43\right.$; the antipode of the this universe is at the redshift 4.93), the probability is very small, $P=0.0002$, and the observed image separation is not compatible with such extreme universe. For a flat universe $\left(\Omega_{m}+\Omega_{\Lambda}=1\right), P=0.56$; the data is not incompatible with a flat universe.

We now perform the same Spearman rank correlation test in $\Omega_{m}$ and $\Omega_{\Lambda}$ parameter space ranging from 0 to 3 . Figure 3 shows the contours of the probability calculated by the Spearman test for each cosmological model. The contours are roughly parallel to the flat universe line $(k=0$, solid line), which illustrates that the mean image separation test can be an independent test for the curvature of the FLRW universe (GPL, PG97). The region above the dashed line has $P<0.05$. Current list of lensed quasars can rule out only the excessively positively curved cosmological models with some statistical significance. Larger number of lensed quasars from future survey will improve the constraints.

\subsection{Likelihood Analysis on Cosmological Parameters}

More flexible statistical test that can incorporate diverse data is the maximum likelihood analysis (Kochanek 1993). The likelihood function can include the probability to be lensed or not as well as to be lensed

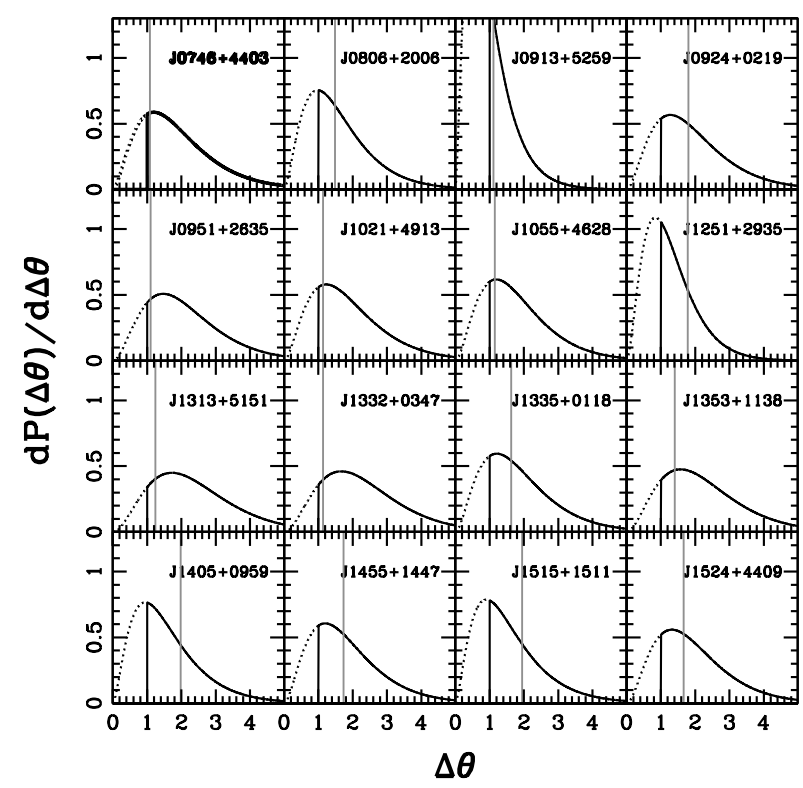

Figure 4. The probability distribution of the angular image separation for each gravitational lens systems in the current concordance model (see Table 1) and the observed image separations (vertical solid line).

into a specific configuration such as the number of images or the image separation (Kochanek 1996). The test for the absolute probability that a given quasar at a given redshift is lensed by a galaxy at a given redshift into a multiple image with given image separation is most common (Kochanek 1996; Chae 2003; Oguri et al. 2008, 2012), which produced familiar constraints on $\Omega_{m}$ and $\Omega_{\Lambda}$, independently confirming the SNe Ia result (Riess et al. 1998; Perlmutter et al. 1999). But calculation of these absolute lensing probability requires the luminosity function of high-redshift quasars and of lens galaxies at all redshift. On the other hand, the likelihood analysis for the relative probability that a quasar at a given redshift is lensed by a galaxy at a given redshift into a given image separation tests if the observed image separations are compatible with a certain cosmological model for a given luminosity function of the lens galaxies. This test, therefore, is less susceptible to the not-well-known quasar luminosity function and its evolution, but the constraints from the test is also less restrictive (Han \& Park 2014). Figure 4 shows the normalized expected probability distribution of the angular image separation for each lensing case (solid curves): under the assumption that the distribution of the velocity dispersion of the lens galaxies is known, the distribution depends only on the cosmology for given quasar and lens galaxy redshifts. The vertical line in each frame shows the actually observed maximum image separation. The likelihood is the product sum of the value of the expected probability at the observed image separation, and represents how much a given cosmological and lens model is consistent with the observed image separations.

In this work, we add one more information not often 


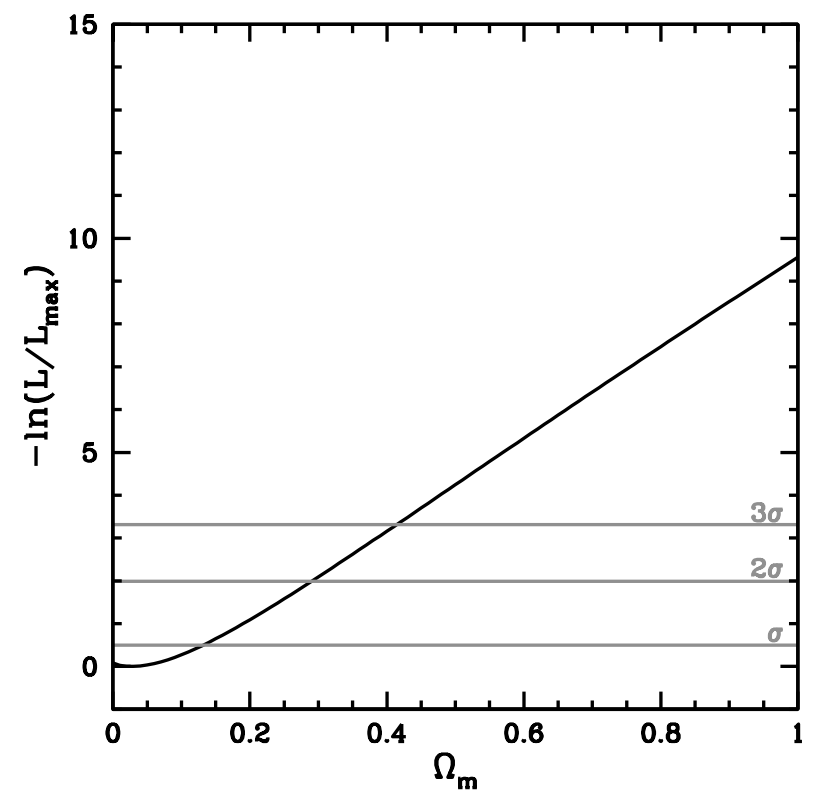

Figure 5. The likelihood of the lensing probability for a flat cosmological model. The solid curve denotes a flat cosmological model with $M_{*, I}=-21.86+5 \log h, \gamma_{\mathrm{FJ}}=3.30$, and $\gamma_{\mathrm{EK}}=-0.31$.

used in the lens probability test: the luminosity of the lens galaxy. Most lensing tests do not utilize the information on the mass of the lens galaxy because mass is not directly observable. Im et al. (1997) used the magnitude of the lens galaxy to infer the critical image separation through Faber-Jackson relation, and tested which cosmological model is most likely for given lens redshifts, source redshifts, and lens magnitudes, yielding the best fit value of $\Omega_{\Lambda}=0.64$ for a flat universe. The number of lens cases used was eight, and they were compiled from very heterogeneous observations.

We repeat the same statistical test to our SQLS sample listed in Table 1. The cosmological model and the probabilities of the lens galaxies are the same as in Han \& Park (2014).

The critical image separation, i.e., the Einstein radius, can be expressed as

$$
\Delta \theta_{\text {crit }}=8 \pi\left(\frac{\sigma_{*}}{c}\right)^{2}\left(\frac{D_{l s}}{D_{o s}}\right) 10^{\frac{4}{5} \frac{1}{\gamma_{\mathrm{FJ}}}\left(M_{*}-M\right)} .
$$

where $\gamma_{\mathrm{FJ}}$ is the index of Faber-Jackson relation, which relates the luminosity to the velocity dispersion. The absolute magnitudes of the lens galaxies with $M$ are corrected for the combined effects of $\mathrm{K}$-correction and luminosity evolution with the term $-2.5 \gamma_{\mathrm{EK}} z_{l}$ (Rusin et al. 2003). The actual observed image separation can differ from the critical image separation due to the observational uncertainty and uncertainty in the theoretical lensing geometry model. Such error is modeled by the Gaussian function (Im et al. 1997). The lensing probability for a given lensed quasar to be observed

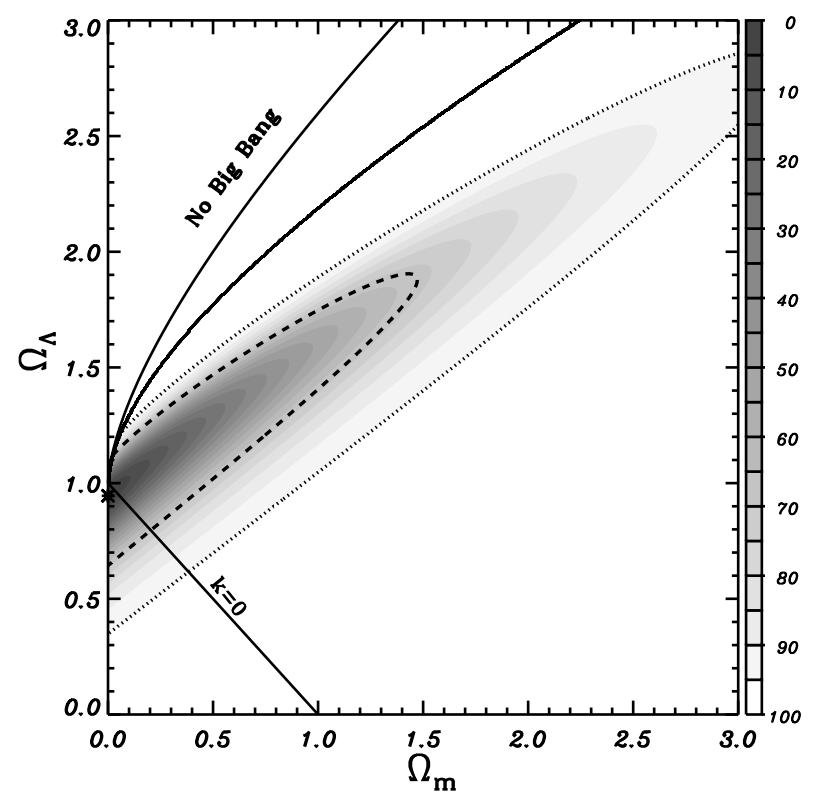

Figure 6. The contours show the likelihood values for cosmological models in $\Omega_{m}$ versus $\Omega_{\Lambda}$ space for the galactic parameters $M_{*, I}=-21.86+5 \log h, \gamma_{\mathrm{FJ}}=3.30$, and $\gamma_{\mathrm{EK}}=-0.31$.

with the image separation $\Delta \theta(i)$ is thus

$$
\begin{array}{r}
P_{i}\left(z_{s}, z_{l}, \Delta \theta, \Omega_{m}, \Omega_{\Lambda}, M_{*}, \sigma_{*}, \gamma_{\mathrm{FJ}}, \gamma_{\mathrm{EK}}\right) \\
\sim G\left(\Delta \theta(i), \Delta \theta_{\mathrm{crit}}, \sigma_{\Delta \theta}\right),
\end{array}
$$

where $G(x, \mu, \sigma)$ is a gaussian function with the mean of $\mu$ and the deviation of $\sigma$. The uncertainty of the image separation, $\sigma_{\Delta \theta}$, is the combined uncertainties of the observed and theoretical image separation, $\sigma_{\Delta \theta}^{2}=$ $\sigma_{\Delta \theta, \text { obs }}^{2}+\sigma_{\Delta \theta, \text { crit }}^{2}\left(\sigma_{\gamma_{\mathrm{FJ}}}, \sigma_{M_{*}}, \sigma_{m_{l}}, \sigma_{\gamma_{\mathrm{EK}}}\right)$. The likelihood function for the whole sample is now

$$
L=\prod_{i} P_{i}\left(z_{s, i}, z_{l, i}, \Delta \theta_{i}, \Omega_{m}, \Omega_{\Lambda}, M_{*}, \sigma_{*}, \gamma_{\mathrm{FJ}}, \gamma_{\mathrm{EK}}\right)
$$

Among our 16 lensed quasars, we exclude SDSS J0913+5259 that shows unreasonably small lensing probability given the observed lens galaxy magnitude. The lens galaxy of SDSS J0913+5259 is not photometrically identified because of the difficulty in the definition of the continuum from it (Oscoz et al. 1997). Either the lensing does not satisfy our assumptions or the information on the lens galaxy is in error.

Rusin et al. (2003) provides the needed galaxy parameters: $M_{*, I}=-21.86+5 \log h \pm 0.29, \gamma_{\mathrm{FJ}}=$ $3.30 \pm 0.59$, and $\gamma_{\mathrm{EK}}=-0.31 \pm 0.21$. When we adopt these values and limit the cosmological model to a flat one, density parameter should be with $95 \%$ confidence $0.0 \leq \Omega_{m} \leq 0.28$ (Figure 5), consistent with the current concordance model. However, the uncertainty in the galaxy parameter is large, especially $\gamma_{\mathrm{FJ}}$ and $\gamma_{\mathrm{EK}}$.

We also extend the likelihood analysis to non-flat cosmological models. Figure 6 shows the likelihood contours in $\Omega_{m}$ versus $\Omega_{\Lambda}$ space. The contours show degeneracy roughly perpendicular to the $\Omega_{m}+\Omega_{\Lambda}=1$ flat 


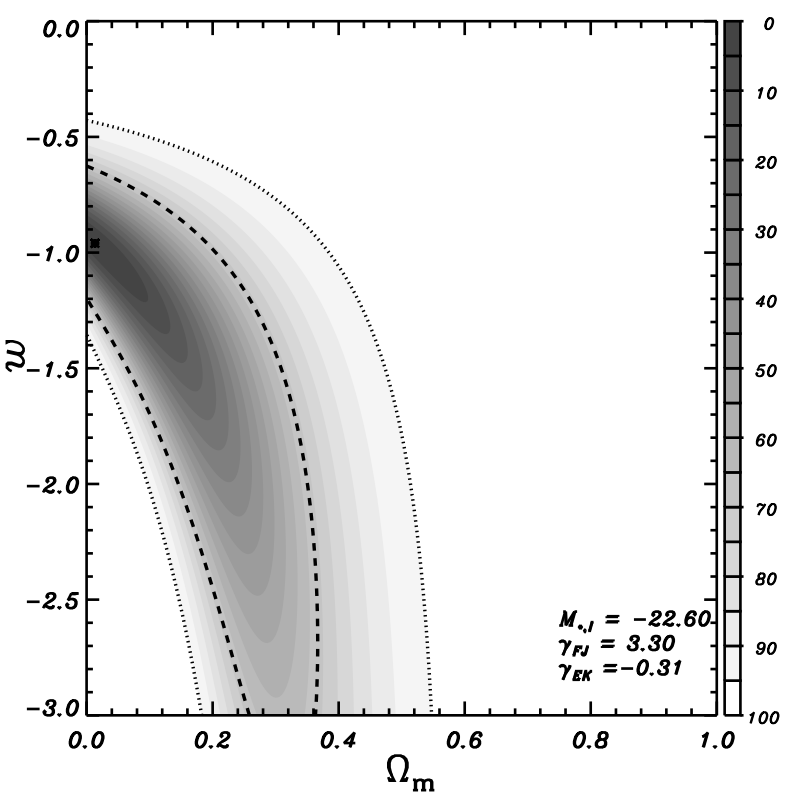

Figure 7. Same as Figure 6 but for the equation of state parameter $w$.

universe, similar to the constraints from SN Ia (Riess et al. 1998; Perlmutter et al. 1999). The maximum likelihood is centered at $\left(\Omega_{m}=0, \Omega_{\Lambda}=0.95\right)$, and $95 \%$ contour (dashed curve) includes flat models with $\Omega_{m}<0.36$. We found that different choice of galactic parameter yields different constraints, often much tighter, which illustrates the constraining power of this test, and yet at the same time also shows that the test is only as good as the knowledge on the galaxy parameters.

The nature of dark energy is one of the most important questions in current science, and any independent constraints on the dark energy equation of state parameter $w$ are desired (Oguri et al. 2012; Cao et al. 2012). Application of the same likelihood to the universe with constant $w$ yields the likelihood contours of Figure 7 for $M_{*, I}=-21.86+5 \log h, \gamma_{\mathrm{FJ}}=3.30$, and $\gamma_{\mathrm{EK}}=-0.31$ : Constraints are very similar to those of Oguri et al. (2012), but $w=-1, \Omega_{m}=0$ model is most likely in our study whereas model with $w=-3$ is most likely in Oguri et al. (2012). The constraints on $w \mathrm{CDM}$ from Planck, WMAP, BAO are consistent with our result (Planck Collaboration, et al. 2014).

\section{Summary and Discussion}

Comparison of the theoretically expected statistical properties of multiply imaged quasars such as the number of lensed quasars and the image separation with those of observed lensed quasars has provided independent constraints on the cosmology. However, the total number of observed lensed quasars is related to the quasar luminosity function at all redshifts and the selection bias during the candidate searching and followup confirmation processes. Here, we used only the image separation of the multiply lensed quasars from the SDSS to constraint the cosmological models or the physical parameters of lens galaxies. Our sample consists of multiply imaged quasars found by SQLS. We first used the expected mean image separation of lensed quasars to test the cosmological models, especially incorporating the selection bias against the lens systems with faint lens galaxy that may fail to be confirmed as real lensed systems due to the magnitude limit of the follow-up observations. This bias becomes more important at higher redshift. Comparison of the expected mean image separations against the maximum image separations of the SQLS lens sample show that the test checks the curvature of the universe, but the constraint is still too weak to reject any non-extreme universe. Our second test is the maximum likelihood analysis that tests the statistics of image separation in 16 SQLS sample that have both the redshifts of quasar and lens galaxy and the magnitude of the lens galaxy reliably measured. The likelihood function incorporates the probability that the observed image separation is realized given the luminosity of the lens galaxy that determines the image separation through velocity-luminosity relation of galaxies with some uncertainties in the same manner as Im et al. (1997). We find that this approach provides very powerful constraints on the cosmological parameters: $0.72 \leq \Omega_{\Lambda} \leq 1.0$ (95\% confidence) for a flat cosmological model and the two-dimensional constraint in $\Omega_{\Lambda}$ versus $\Omega_{m}$ space is comparable to the constraints from more complex lens probability tests. However, we caution that this test is only as powerful as the accuracy of the relevant galactic parameters, such as the luminosity of typical galaxies, k-correction parameter, and the Faber-Jackson slope. As the number of multiple image gravitationally lensed quasars grow with future surveys, the image separation statistics will provide more robust constraints on the cosmological parameters.

\section{ACKNOWLEDGMENTS}

This research was supported by Basic Science Research Program through the National Research Foundation of Korea (NRF) funded by the Ministry of Education, Science and Technology (2012R1A1A4A01013596). DHH also acknowledges the support of Brain Korea 21 Plus Program through the National Research Foundation of Korea funded by the Ministry of Education, Science and Technology.

\section{REFERENCES}

Ahn, C. P., et al. 2014, The Tenth Data Release of the Sloan Digital Sky Survey: First Spectroscopic Data from the SDSS-III Apache Point Observatory Galactic Evolution Experiment, ApJS, 211, 17

Browne, I. W. A., et al. 2003, The Cosmic Lens All-Sky Survey - II. Gravitational Lens Candidate Selection and Follow-Up, MNRAS, 341, 13

Cao, S., Zhu, Z.-H., \& Zhao, R. 2011, Testing and Selecting Dark Energy Models with Lens Redshift Data, PhRvD, 84,023005 
Cao, S., \& Zhu, Z.-H. 2012, Constraints on Cosmological Models from Lens Redshift Data, A\&A, 538, A43

Chae, K.-H. 2003, The Cosmic Lens All-Sky Survey: Statistical Strong Lensing, Cosmological Parameters, and Global Properties of Galaxy Populations, MNRAS, 346, 746

Chae, K.-H. 2010, Galaxy Evolution from Strong-Lensing Statistics: the Differential Evolution of the Velocity Dispersion Function in Concord with the $\Lambda$ Cold Dark Matter Paradigm, MNRAS, 402, 2031

Chiba, M., \& Yoshii, Y. 1997, Do Lensing Statistics Rule Out a Cosmological Constant?, ApJ, 489, 485

Chiba, M., \& Yoshii, Y. 1999, New Limits on a Cosmological Constant from Statistics of Gravitational Lensing, ApJ, 510,42

Choi, Y.-Y., Park, C., \& Vogeley, M. S. 2007, Internal and Collective Properties of Galaxies in the Sloan Digital Sky Survey, ApJ, 658, 884

Croom, S. M., et al. 2009, The 2dF-SDSS LRG and QSO Survey: the QSO Luminosity Function at $0.4<z<2.6$, MNRAS, 399, 1755

Eigenbrod, A., et al. 2006, COSMOGRAIL: the COSmological MOnitoring of GRAvItational Lenses. II. SDSS J0924+0219: the Redshift of the Lensing Galaxy, the Quasar Spectral Variability and the Einstein Rings, A\&A, 451, 747

Eigenbrod, A., et al. 2006, COSMOGRAIL: the COSmological MOnitoring of GRAvItational Lenses. III. Redshift of the Lensing Galaxy in Eight Gravitationally Lensed Quasars, A\&A, 451, 759

Eigenbrod, A., Courbin, F., \& Meylan, G. 2007, COSMOGRAIL: the COSmological MOnitoring of GRAvItational Lenses. VI. Redshift of the Lensing Galaxy in Seven Gravitationally Lensed Quasars, A\&A, 465, 51

Fukugita, M., et al. 1992, Statistical Properties of Gravitational Lenses with a Nonzero Cosmological Constant, ApJ, 393, 3

Fukugita, M., \& Turner, E. L. 1991, Gravitational Lensing Frequencies - Galaxy Cross-Sections and Selection Effects, MNRAS, 253, 99

Gott, J. R., III, Park, M.-G., \& Lee, H. M. 1989, Setting Limits on q0 from Gravitational Lensing, ApJ, 338, 1

Han, D.-H., \& Park, M.-G. 2014, Constraining Cosmology with Image Separation Statistics for GravitationallyLensed Quasars in the Sloan Digital Sky Survey, JKPS, 65,827

Helbig, P. 1998, The $\Delta \theta-z_{s}$ Relation for Gravitational Lenses as a Cosmological Test, MNRAS, 298, 395

Im, M., Griffiths, R. E., \& Ratnatunga, K. U. 1997, A Measurement of the Cosmological Constant Using Elliptical Galaxies as Strong Gravitational Lenses, ApJ, 475, 457

Inada, N., et al. 2003, SDSS J092455.87+021924.9: An Interesting Gravitationally Lensed Quasar from the Sloan Digital Sky Survey, AJ, 126, 666

Inada, N., et al. 2006, SDSS J0806+2006 and SDSS J1353+1138: Two New Gravitationally Lensed Quasars from the Sloan Digital Sky Survey, AJ, 131, 1934

Inada, N., et al. 2007, Two New Gravitationally Lensed Double Quasars from the Sloan Digital Sky Survey, AJ, 133,206

Inada, N., et al. 2010, The Sloan Digital Sky Survey Quasar Lens Search. IV. Statistical Lens Sample from the Fifth Data Release, AJ, 140, 403

Inada, N., et al. 2012, The Sloan Digital Sky Survey Quasar Lens Search. V. Final Catalog from the Seventh Data
Release, AJ, 143, 119

Jackson, N., et al. 2012, New Lensed Quasars from the MUSCLES Survey, MNRAS, 419, 2014

Kayo, I., et al. 2007, A New Quadruply Lensed Quasar: SDSS J125107.57+293540.5, AJ, 134, 1515

Kayo, I., et al. 2010, Eight New Quasar Lenses from the Sloan Digital Sky Survey Quasar Lens Search, AJ, 139, 1614

Kochanek, C. S. 1993, Analytic Results for the Gravitational Lens Statistics of Singular Isothermal Spheres in General Cosmologies, MNRAS, 261, 453

Kochanek, C. S. 1996, Is There a Cosmological Constant?, ApJ, 466, 638

Kochanek, C. S., Falco, E. E., \& Muñoz, J. A. 1999, Why Quasar Pairs Are Binary Quasars and Not Gravitational Lenses, ApJ, 510, 590

Komatsu, E., et al. 2009, Five-Year Wilkinson Microwave Anisotropy Probe Observations: Cosmological Interpretation, ApJS, 180, 330

Lee, H.-A., \& Park, M.-G. 1994, Statistical Properties of Gravitational Lensing in Cosmological Models with Cosmological Constant, JKAS, 27, 103

Maoz, D., \& Rix, H.-W. 1993, Early-Type Galaxies, Dark Halos, and Gravitational Lensing Statistics, ApJ, 416, 425

Morokuma, T., et al. 2007, Discovery of a Gravitationally Lensed Quasar from the Sloan Digital Sky Survey: SDSS J133222.62+034739.9, AJ, 133, 214

Myers, S. T., et al. 2003, The Cosmic Lens All-Sky Survey - I. Source Selection and Observations, MNRAS, 341, 1

Ofek, E. O., et al. 2007, SDSS J131339.98+515128.3: a New Gravitationally Lensed Quasar Selected Based on NearInfrared Excess, MNRAS, 382, 412

Oguri, M., et al. 2004, SDSS J1335+0118: A New TwoImage Gravitational Lens, PASJ, 56, 399

Oguri, M., et al. 2006, The Sloan Digital Sky Survey Quasar Lens Search. I. Candidate Selection Algorithm, AJ, 132, 999

Oguri, M., et al. 2008, The Sloan Digital Sky Survey Quasar Lens Search. III. Constraints on Dark Energy from the Third Data Release Quasar Lens Catalog, AJ, 135, 512

Oguri, M., et al. 2012, The Sloan Digital Sky Survey Quasar Lens Search. VI. Constraints on Dark Energy and the Evolution of Massive Galaxies, AJ, 143, 120

Oscoz, A., et al. 1997, Support for the Gravitational Lens Interpretation of SBS 0909+532, ApJ, 491, L7

Park, M.-G. 1996, Gravitational Lensing Statistics in a Flat Universe, JKPS, 29, 664

Park, M.-G., \& Gott, J. R. III. 1997, Curvature of the Universe and Observed Gravitational Lens Image Separations versus Redshift, ApJ, 489, 476

Percival, W. J., et al. 2007, Measuring the Baryon Acoustic Oscillation scale using the Sloan Digital Sky Survey and 2dF Galaxy Redshift Survey, MNRAS, 381, 1053

Perlmutter, S., et al. 1997, Measurements of the Cosmological Parameters $\Omega$ and $\Lambda$ from the First Seven Supernovae at $\mathrm{z} \geq 0.35$, ApJ, 483, 565

Perlmutter, S., et al. 1999, Measurements of $\Omega$ and $\Lambda$ from 42 High-Redshift Supernovae, ApJ, 517, 565

Pindor, B., et al. 2006, SDSS J102111.02+491330.4: A Newly Discovered Gravitationally Lensed Quasar, AJ, 131,41

Planck Collaboration, et al. 2014, Planck 2013 results. XVI. Cosmological parameters, A\&A, 571, A16

Riess, A. G., et al. 1998, Observational Evidence from Su- 
pernovae for an Accelerating Universe and a Cosmological Constant, AJ, 116, 1009

Ross, N. P., et al. 2013, The SDSS-III Baryon Oscillation Spectroscopic Survey: The Quasar Luminosity Function from Data Release Nine, ApJ, 773, 14

Rusin, D., et al. 2003, The Evolution of a Mass-Selected Sample of Early-Type Field Galaxies, ApJ, 587, 143

Schechter, P. L., et al. 1998, The First FIRST Gravitationally Lensed Quasar: FBQ 0951+2635, AJ, 115, 1371

Schneider, P., Ehlers, J., \& Falco, E. E. 1992, Gravitational Lenses (Berlin: Springer)

Schneider, D. P., et al. 2005, The Sloan Digital Sky Survey Quasar Catalog. III. Third Data Release, AJ, 130, 367

Schneider, D. P., et al. 2007, The Sloan Digital Sky Survey Quasar Catalog. IV. Fifth Data Release, AJ, 134, 102

Schneider, D. P., et al. 2010, The Sloan Digital Sky Survey Quasar Catalog. V. Seventh Data Release, AJ, 139, 2360
Sheth, R. K., et al. 2003, The Velocity Dispersion Function of Early-Type Galaxies, ApJ, 594, 225

Singal, J., et al. 2013, The Radio and Optical Luminosity Evolution of Quasars. II. The SDSS Sample, ApJ, 764, 43

Turner, E. L., Ostriker, J. P., \& Gott, J. R. III. 1984, The Statistics of Gravitational Lenses - The Distributions of Image Angular Separations and Lens Redshifts, ApJ, 284, 1

Walsh, D., Carswell, R. F., \& Weymann, R. J. 1979, 0957 +561 A, B - Twin Quasistellar Objects or Gravitational Lens, Nature, 279, 381

Williams, L. L. R. 1997, Image Separation versus Redshift of Lensed QSOs - Implications for Galaxy Mass Profiles, MNRAS, 292, L27

York, D. G., et al. 2000, The Sloan Digital Sky Survey: Technical Summary, AJ, 120, 1579 